\title{
A Incontinência Urinária em Mulheres Praticantes de Exercício Recreativo: Um Estudo Transversal
}

\author{
Urinary Incontinence in Women Who Practice \\ Recreational Exercise: A Cross-Sectional Study
}

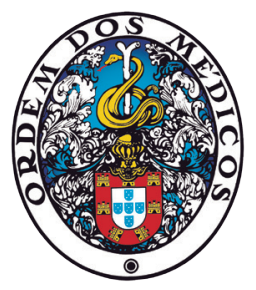

\author{
Maria Francisca SILVA $₫ 1$, Rui PRADO COSTA ${ }^{2}$, Carla Maria OLIVEIRA ${ }^{3,4,5}$, Susana MOREIRA ${ }^{2}$ \\ Acta Med Port 2021 Nov;34(11):724-732 - https://doi.org/10.20344/amp.14004
}

\begin{abstract}
RESUMO
Introdução: A incontinência urinária afeta até um terço das mulheres em Portugal. O exercício pode ser fator precipitante/de exacerbação, mas também protetor, se for adequado. O objetivo deste estudo é determinar a prevalência de incontinência urinária em mulheres praticantes de exercício recreativo, avaliar a relação com o tipo de exercício e outros fatores de risco coexistentes, e aferir se o tema é abordado nos ginásios.
\end{abstract}

Material e Métodos: Estudo transversal através de questionários de auto-preenchimento em ginásios do Porto e Vila Nova de Gaia. Resultados: Duzentas e noventa mulheres completaram os questionários. Destas, $67,6 \%$ tinham menos de 40 anos, $25,2 \%$ referiam incontinência e 53,4\% tinham pelo menos um fator de risco não relacionado com exercício. Verificou-se uma associação estatisticamente significativa $(p<0,05)$ entre incontinência, obesidade e obstipação. Exercícios de alto impacto eram incluídos nos treinos de $62,1 \%$ das mulheres continentes e $50,9 \%$ das incontinentes. O tema incontinência e fortalecimento muscular do pavimento pélvico foi abordado em apenas $5,5 \%$ na avaliação inicial, 9,7\% em treinos coletivos e em 13,5\% das 37 mulheres com treinos individuais.

Discussão: A maior proporção de mulheres continentes, comparativamente às incontinentes, que praticavam exercício extenuante, sugere que este era um fator provocativo para algumas, não se verificando, contudo, uma associação estatisticamente significativa entre incontinência e tipo de exercício.

Conclusão: A incontinência urinária afeta mulheres praticantes de exercício recreativo, independentemente da idade e características do exercício. É raramente abordada nos ginásios, sendo necessário sensibilizar os profissionais para potenciar os efeitos preventivos/ terapêuticos do exercício na função do pavimento pélvico e no controlo de fatores de risco modificáveis.

Palavras-chave: Desporto; Exercício; Incontinência Urinária

\section{ABSTRACT}

Introduction: Urinary incontinence affects up to one third of women in Portugal. Exercise can be a precipitating/aggravating factor but also protective, if appropriate. The aim of this study was to determine the prevalence of urinary incontinence in women who practice recreational exercise, to assess its relationship with the type of exercise and other coexisting risk factors, and assess whether the topic is addressed in gyms.

Material and Methods: Cross-sectional study using self-reported questionnaires in gyms in Porto and Vila Nova de Gaia, Portugal. Results: Two hundred and ninety women completed the questionnaires. From these, $67.6 \%$ were under 40 years old, $25.2 \%$ reported incontinence, and $53.4 \%$ had at least one risk factor unrelated to exercise. There was a statistically significant association $(p<0.05)$ between incontinence, obesity and constipation. High impact exercises were included in the training of $62.1 \%$ continent and $50.9 \%$ incontinent women. The topic of incontinence and strengthening of the pelvic floor muscles was addressed in only $5.5 \%$ in the initial assessment, $9.7 \%$ in collective training, and in $13.5 \%$ of the 37 women with individualized training.

Discussion: The higher proportion of continent women - compared to incontinent - who practiced strenuous exercise suggests that this might be a provocative factor for some, although there was no statistically significant association between incontinence and type of exercise.

Conclusion: Urinary incontinence affects women who practice recreational exercise, regardless of age and exercise characteristics. It is rarely addressed in gyms, and it is necessary to raise the awareness of professionals to enhance the preventive/therapeutic effects of exercise on the function of the pelvic floor and in the control of modifiable risk factors.

Keywords: Exercise; Sports; Urinary Incontinence

\section{INTRODUÇÃO}

A incontinência urinária (IU) é definida pela International Continence Society (ICS) como a "queixa de perda involuntária de urina". ${ }^{1}$ É difícil estabelecer a sua prevalência real, uma vez que a definição de IU, as características das populações e metodologias divergem entre os estudos, sendo reportados valores entre $25 \%$ e $45 \%$, nas mulheres acima dos 20 anos, não gravidas. ${ }^{2}$ Um estudo realizado em
2009 na população portuguesa revelou que a prevalência de IU nas mulheres com mais de 40 anos era de $21,4 \%$, sendo a IU de esforço (IUE) a mais frequente $(42,2 \%)^{3}$ Recentemente, uma nova investigação aponta para uma prevalência de $35,1 \%$ entre mulheres dos 19 aos 90 anos, mantendo-se a IUE como a mais frequente $(76,2 \%) .{ }^{4}$ Apesar desta elevada prevalência, apenas $25 \%$ a $61 \%$ procura

1. Faculdade de Medicina. Universidade do Porto. Porto. Portugal.

2. Serviço de Medicina Física e Reabilitação. Centro Hospitalar Universitário de São João. Porto. Portugal.

3. i3S - Instituto de Investigação e Inovação em Saúde. Universidade do Porto. Porto. Portugal.

4. INEB - Instituto de Engenharia Biomédica. Universidade do Porto. Porto. Portugal.

5. ESS-IPP - Escola Superior da Saúde do Porto. Instituto Politécnico do Porto. Porto. Portugal.

$\triangle$ Autor correspondente: Maria Francisca Silva. franciscacrpsilva@gmail.com

Recebido: 28 de abril de 2020 - Aceite: 07 de julho de 2020 - Online issue published: 02 de novembro de 2021

Copyright @ Ordem dos Médicos 2021 
tratamento. $^{2}$

A IU pode ser uma consequência de disfunção do pavimento pélvico. Para existir continência urinária é necessário que a pressão intra-vesical seja inferior à pressão intra-uretral e, portanto, qualquer fator que leve ao aumento da primeira face à segunda pode ser causa da IU. $.^{5,6} \mathrm{Di}-$ versos fatores podem contribuir para a ocorrência de IU, nomeadamente paridade, parto vaginal, idade avançada, menopausa e situações que condicionam aumento significativo e/ou crónico da pressão intra-abdominal (PIA) como obesidade, tosse crónica e esforços que impliquem cargas elevadas. . $^{2,3,7,8}$

Não sendo uma condição ameaçadora de vida, a IU tem um impacto negativo importante na qualidade de vida, levando muitas vezes ao isolamento social e a quadros de depressão e ansiedade. Os episódios de perda urinária interferem com as tarefas do dia-a-dia bem como com a atividade profissional, a vida sexual, e a prática desportiva e de atividades de lazer. . $^{7,10}$

A IU é uma importante barreira à prática de exercício físico e pode levar ao seu abandono, ${ }^{11,12}$ privando as doentes dos benefícios reconhecidos na manutenção de uma vida saudável, na melhoria do prognóstico de múltiplas doenças (ex. diabetes mellitus, hipertensão arterial, dislipidemia, obesidade) e na promoção da saúde mental. ${ }^{13}$ De facto, a Organização Mundial de Saúde considera a inatividade física como o quarto fator de risco para a mortalidade mundial e aconselha que toda a população se mantenha fisicamente ativa. ${ }^{13}$

Se por um lado a atividade e o exercício físico devem ser encorajados, por outro, o seu efeito sobre o pavimento pélvico e a IU não está completamente esclarecido. ${ }^{14,15} \mathrm{Du}$ rante o exercício, vários são os momentos em que o equilíbrio entre as pressões intra-vesical e intra-uretral pode estar comprometido, por efeito das forças de reação do solo e do aumento da PIA, o que pode precipitar ou exacerbar a IU. A própria definição de IUE, como a "queixa de perda involuntária de urina durante o esforço físico (como por exemplo atividade desportiva, tosse ou espirro)", alerta-nos para a forte relação que existe entre o esforço físico e as perdas urinárias.

Em 1994, Bø K et al ${ }^{16}$ demonstrou que a contração dos músculos da região abdominal, dos adutores da coxa ou dos glúteos produzia uma contração sinérgica nos músculos do pavimento pélvico (MPP). Mais tarde, em 2004, a mesma autora sugeriu duas hipóteses para explicar o efeito do exercício nos MPP: (1) o aumento da PIA e a co-contração dos MPP durante o exercício físico condiciona um fortalecimento e hipertrofia dos MPP, elevando o plano da uretra e dos órgãos pélvicos, o que reduz o risco de IU; (2) o exercício extenuante ou com levantamento de grandes cargas produz aumentos significativos, repetitivos da PIA, aumenta o stress sobre o pavimento pélvico e condiciona o seu enfraquecimento. ${ }^{17}$ Há evidências que apoiam ambas as teorias, ${ }^{14,15}$ ainda que esteja melhor estudada a relação da IU com o exercício extenuante ou de alto impacto do que com o exercício leve a moderado. ${ }^{14}$
Tanto quanto sabemos, os estudos epidemiológicos sobre IU em Portugal são escassos, não existindo nenhum estudo cuja população alvo seja a população portuguesa feminina praticante de exercício físico recreativo, e que avalie mulheres de diferentes escalões etários. Sendo o exercício físico um fator modificável, é essencial entender a relação entre a atividade física, a disfunção do pavimento pélvico e a IU, com vista à prevenção da IU e à diminuição das taxas de abandono da prática desportiva.

O objetivo primário deste trabalho consiste no estudo da prevalência e caracterização da IU em mulheres praticantes de exercício físico recreativo em ginásios e na avaliação da sua relação com características demográficas e fatores de risco conhecidos, nomeadamente o tipo de exercício praticado. O objetivo secundário consiste na aferição da abordagem da temática da IU e dos potenciais efeitos do exercício em diferentes momentos da prática de exercício físico em ginásios (avaliação física, treinos coletivos e individuais).

\section{MATERIAL E MÉTODOS}

Efetuamos um estudo transversal observacional com recolha de um questionário em papel, em nove ginásios dos concelhos do Porto e de Vila Nova de Gaia (Portugal), durante os meses de janeiro e fevereiro de 2020. Foram incluídas apenas mulheres com idade superior a 18 anos e que frequentavam o ginásio pelo menos uma vez por semana. A seleção da amostra foi efetuada por conveniência, tendo sido solicitada a colaboração das participantes à chegada ao ginásio.

Desenhámos um inquérito anónimo de auto-preenchimento (ver Apêndice 1: https://www.actamedicaportuguesa.com/revista/index.php/amp/article/view/14004/Apendice_01.pdf)), constituído principalmente por questões fechadas, tendo como referência o questionário de McKenzie et a $/^{18}$ e o International Consultation on Incontinence Modular Questionnaire - Urinary Incontinence Short Form (ICIQ-UI $\mathrm{SF}$ ) validado para português. ${ }^{19} \mathrm{O}$ inquérito foi estruturado em seis secções: dados gerais (idade, peso, altura, escolaridade), antecedentes médicos, antecedentes ginecológicos e obstétricos, atividade física, incontinência urinária e exercícios dos músculos do pavimento pélvico [pelvic floor muscle training (PFMT)]. O questionário contemplou ainda perguntas relativas à interferência da IU com a prática do exercício, ao conhecimento sobre PFMT, à abordagem da temática da IU e PFMT no ginásio em algum momento (avaliação física, treino de grupo ou individual) e à discussão deste problema de saúde com o médico assistente.

Incluímos os seguintes fatores considerados de ris$\mathrm{CO}^{2,3,7,8}$ : menopausa e terapêutica hormonal de substituição, obesidade, paridade e via de parto, cirurgias genito-urinárias, tratamentos ou lesões da zona pélvica, tipo de exercício físico praticado, doenças crónicas (diabetes, depressão, hipertensão, tosse crónica, obstipação e acidente vascular cerebral), ser atleta de alta competição e levantar pesos de elevada carga.

A caracterização da IU foi realizada através do ICIQ-UI 
SF, tendo-se considerado como 'incontinentes' as participantes que reportaram perdas urinárias nas últimas quatro semanas em relação à data de preenchimento do questionário. Para determinar a gravidade, recorremos à classificação proposta por Klovning et al, que correlaciona o ICIQ-UI SF com o Incontinence Severity Index. ${ }^{20}$

Para a caracterização do tipo de exercício físico praticado, consideramos duas categorias: (1) exercício fatigante com aumento significativo da PIA (como o levantamento repetido de cargas elevadas) e/ou de alto impacto; e (2) exercícios de baixo impacto. No primeiro grupo foram incluídas as aulas de Body Attack, Body Combat, Body Pump, Power Jump, Step, Cross Fit, total condicionamento e circuito/ funcional, assim como treino nas máquinas ou com per- sonal trainer, (PT) maioritariamente musculação com levantamento de cargas elevadas e outras atividades como corrida. A categoria de baixo impacto incluiu as aulas de Body balance, Yoga, Pilates, Hidroginástica, Core/CXWor", Cycling/RPM, localizada e Zumba, assim como nadar na piscina, treino nas máquinas ou com $\mathrm{PT}$, maioritariamente musculação sem levantamento de cargas elevadas, e outras atividades como caminhadas ou dança.

O tamanho da amostra foi estimado assumindo uma prevalência esperada de $21,4 \%$ para a $I^{3}$ e um nível de significância de 5\%. Um tamanho de amostra de 259 muIheres foi considerado o necessário para atingir um nível de precisão de $5 \%$.

Tabela 1 - Associação univariada e multivariada entre a incontinência urinária e os fatores de risco

\begin{tabular}{|c|c|c|c|c|c|c|}
\hline & $\begin{array}{c}\text { Continentes } \\
n=217\end{array}$ & $\begin{array}{c}\text { Incontinentes } \\
n=73^{+}\end{array}$ & $\begin{array}{l}\text { OR não ajustado } \\
\text { (IC 95\%) }\end{array}$ & $p$ & $\begin{array}{l}\text { OR ajustado } \\
\text { (IC } 95 \%)\end{array}$ & $p$ \\
\hline Idade (anos) & $31(26 ; 41,5)$ & $34,5(27 ; 46)$ & $1,01(0,99-1,03)$ & 0,184 & $0,99(0,95-1,03)$ & 0,691 \\
\hline \multicolumn{7}{|l|}{ Idade } \\
\hline 18 - 39 anos & $152(70,4)$ & $44(60,3)$ & Ref & & & \\
\hline $40-59$ anos & $50(23,1)$ & $24(32,9)$ & $1,66(0,92-3,00)$ & 0,094 & & \\
\hline$\geq 60$ anos & $14(6,5)$ & $5(6,8)$ & $1,23(0,42-3,61)$ & 0,702 & & \\
\hline \multicolumn{7}{|l|}{ Escolaridade } \\
\hline $4^{\mathrm{a}}$ classe & $6(2,8)$ & $1(1,4)$ & Ref & & & \\
\hline $9^{\circ}$ ano & $5(2,3)$ & $1(1,4)$ & $1,20(0,06-24,47)$ & 0,906 & & \\
\hline $12^{\circ}$ ano & $21(9,7)$ & $12(36,4)$ & $3,43(0,37-31,97)$ & 0,279 & & \\
\hline Curso superior & $184(85,2)$ & $58(80,6)$ & $1,92(0,23-16,31)$ & 0,548 & & \\
\hline \multicolumn{7}{|l|}{ IMC } \\
\hline$<25 \mathrm{~kg} / \mathrm{m}^{2}$ & $174(80,6)$ & $46(63,9)$ & Ref & & Ref & \\
\hline$\geq 25 \mathrm{~kg} / \mathrm{m}^{2}$ & $42(19,4)$ & $26(36,1)$ & $2,34(1,30-4,21)$ & $0,005^{*}$ & $2,18(1,16-4,09)$ & $0,015^{*}$ \\
\hline \multicolumn{7}{|l|}{ Obstipação } \\
\hline Não & $205(94,5)$ & $57(78,1)$ & Ref & & Ref & \\
\hline Sim & $12(5,5)$ & $16(21,9)$ & $4,80(2,15-10,71)$ & $<0,001^{*}$ & $4,91(2,06-11,68)$ & $<0,001^{*}$ \\
\hline \multicolumn{7}{|c|}{ Cirurgia genito-urinária } \\
\hline Não & $195(96,1)$ & $59(89,4)$ & Ref & & & \\
\hline Sim & $8(3,9)$ & $7(10,6)$ & $2,89(1,01-8,31)$ & $0,049^{*}$ & & \\
\hline \multicolumn{7}{|l|}{ Menopausa } \\
\hline Não & $185(86,7)$ & $59(81,9)$ & Ref & & & \\
\hline $\operatorname{Sim}$ & $29(13,6)$ & $13(18,1)$ & $1,41(0,69-2,88)$ & 0,352 & $0,74(0,21-2,55)$ & 0,632 \\
\hline \multicolumn{7}{|c|}{ Terapia hormonal de substituição } \\
\hline Não & $211(97,2)$ & $71(97,3)$ & Ref & & & \\
\hline Sim & $6(2,8)$ & $2(2,7)$ & $0,99(0,20-5,02)$ & 0,991 & & \\
\hline \multicolumn{7}{|c|}{ Paridade ( $n^{\circ}$ gestações) } \\
\hline 0 & $161(74,2)$ & $45(61,6 \%)$ & Ref & & Ref & \\
\hline$\geq 1$ & $56(25,8)$ & $28(38,4)$ & $1,79(1,02-3,14)$ & $0,042^{*}$ & $1,57(0,57-4,32)$ & 0,381 \\
\hline \multicolumn{7}{|l|}{ Partos vaginais } \\
\hline 0 & $183(84,3)$ & $56(76,7)$ & Ref & & Ref & \\
\hline$\geq 1$ & $34(15,7)$ & $17(23,3)$ & $1,63(0,85-3,14)$ & 0,142 & $1,49(0,53-4,15)$ & 0,450 \\
\hline
\end{tabular}

Os dados são apresentados como mediana $\left(1^{\circ} \mathrm{Q} ; 3^{\circ} \mathrm{Q}\right)$, contagem (\%) ou OR (razão de chances - odds ratio)

† foram incluídos todos os tipos de incontinência urinária

* estatisticamente significativo considerando nível de significância de 0,05.

IMC: índice de massa corporal; Ref: grupo de referência 
A estatística descritiva foi usada para caracterizar a população, sendo que as variáveis contínuas foram sumariadas pela mediana (primeiro e terceiro quartil) e as variáveis categóricas pela contagem (percentagem) em cada categoria. A medida usada para estabelecer a associação entre a IU e os fatores de risco foi o odds ratio. O odds ratio é o rácio entre o odds de ser IU no grupo dos expostos (ex. ter obstipação) e o odds de ser IU no grupo dos não expostos (ex. não ter obstipação). A regressão logística uni- e multivariável foi usada para estimar o odds ratio não ajustado e ajustado, respetivamente, para possíveis variáveis confundidoras. Os fatores de risco apenas foram incluídos na regressão multivariável quando estatisticamente significativos na regressão univariável ou fortemente associados pela literatura. O teste de Hosmer-Lemeshow foi usado para avaliar o ajuste do modelo. O subtipo de IU de urgência (IUU) foi excluído da análise dos parâmetros relacionados com a prática de exercício, devido à improbabilidade da sua relação fisiopatológica.

Para efeitos de tratamento estatístico, todos os inquéritos foram considerados para análise, mesmo os que não foram respondidos na totalidade. Para cada variável, os cálculos foram efetuados excluindo os casos omissos (situação de não resposta à pergunta referente do questionário) e foi utilizada a 'percentagem válida' na análise descritiva.

A análise estatística dos dados foi realizada recorrendo ao software IMB SPSS Statistics 26 . Os testes estatísticos foram considerados bilaterais e a significância estatística foi considerada com um valor de $p<0,05$.

Este estudo foi aprovado pela Comissão de Ética para a Saúde do Centro Hospitalar Universitário São João.

\section{RESULTADOS}

Duzentas e noventa mulheres completaram os questio- nários. Em relação à totalidade da amostra ( $n=290)$, a mediana de idades foi de $31(26 ; 43)$ anos, com um mínimo de 18 e um máximo de 80 anos. A maioria tinha uma licenciatura ou grau superior $(84,1 \%, n=243)$. A mediana relativa ao período de prática de exercício regular foi de 26 $(12 ; 84)$ meses, com um mínimo de um mês e um máximo de 480 meses. Obtivemos uma prevalência de incontinência de $25,2 \%(n=73)$, sendo que a IUE foi a mais prevalente $(13,6 \%, n=39)$, seguindo-se a IU mista $(5,6 \%, n=$ 16) e a IUU $(5,2 \%, n=15)$. Apenas $28,6 \%(n=20)$ das mulheres incontinentes tinha reportado este problema ao seu médico. A análise descritiva comparativa entre os grupos, continentes e incontinentes, encontra-se representada na Tabela 1 (características sociodemográficas, fatores de risco para IU) e na Tabela 2 (variáveis relacionadas com o exercício físico, IU). Cento e cinquenta e cinco mulheres $(53,4 \%)$ tinham pelo menos um fator de risco para IU não relacionado com o exercício: $71,2 \%(n=52)$ das incontinentes e $47,5 \%$ ( $n=103)$ das continentes. Não foram encontradas diferenças estatisticamente significativas entre o tipo de exercício praticado, a frequência de treinos semanais, o levantamento de cargas elevadas e antecedentes de prática desportiva profissional ou de alta competição, como se pode observar pelos valores de $p$ da Tabela 2 .

$\mathrm{Na}$ análise não ajustada, observou-se uma associação significativa entre a IU e as variáveis IMC, obstipação, antecedentes de cirurgia genito-urinária e paridade, sendo que, quando ajustado, apenas se mantiveram significativas as variáveis obstipação e IMC, como se pode verificar pelos valores- $p$ do odds ratio não ajustado e ajustado da Tabela 1. A presença de obstipação aumenta cerca de cinco vezes o odds de desenvolvimento de IU em praticantes de exercício; ser obeso ou pré-obeso aumenta o risco em cerca de duas vezes (Obstipação, OR = 4,91, 95\% IC 2,06 - 11,68; IMC $\geq 25 \mathrm{~kg} / \mathrm{m}^{2}$ vs $<25 \mathrm{~kg} / \mathrm{m}^{2}, \mathrm{OR}=2,18,95 \%$ IC

Tabela 2 - Associação univariada entre a incontinência urinária de esforço ou mista e o exercício físico

\begin{tabular}{|c|c|c|c|c|}
\hline & $\begin{array}{c}\text { Continentes } \\
n=217\end{array}$ & $\begin{array}{c}\text { Incontinentes } \\
n=55^{\dagger}\end{array}$ & $\begin{array}{l}\text { OR não ajustado } \\
\text { (IC 95\%) }\end{array}$ & $p$ \\
\hline Período de prática de exercício regular (meses) & $26(12 ; 84)$ & $36(12 ; 120)$ & $1,00(1,00-1,01)$ & 0,084 \\
\hline \multicolumn{5}{|l|}{ Tipo de exercício } \\
\hline Exclusivamente baixo impacto & $77(37,9)$ & $26(49,1)$ & Ref & \\
\hline Alto impacto/fatigante ou ambos & $126(62,1)$ & $27(50,9)$ & $0,64(0,35-1,17)$ & 0,143 \\
\hline \multicolumn{5}{|l|}{ Frequência de treinos } \\
\hline 1 ou 2 por semana & $19(10,6)$ & $7(14,6)$ & Ref & \\
\hline$\geq 3$ por semana & $160(89,4)$ & $41(85,4)$ & $0,70(0,27-1,77)$ & 0,445 \\
\hline \multicolumn{5}{|l|}{ Levantamento de cargas pesadas } \\
\hline Não & $126(60,9)$ & $36(67,9)$ & Ref & \\
\hline Sim & $81(39,1)$ & $17(32,1)$ & $0,74(0,39-1,39)$ & 0,345 \\
\hline \multicolumn{5}{|l|}{ Atleta de alta competição } \\
\hline Não & $209(96,3)$ & $51(92,7)$ & Ref & \\
\hline $\operatorname{Sim}$ & $8(3,7)$ & $4(7,3)$ & $2,05(0,59-7,07)$ & 0,256 \\
\hline
\end{tabular}

Os dados são apresentados como mediana $\left(1^{\circ} \mathrm{Q} ; 3^{\circ} \mathrm{Q}\right)$, contagem $(\%)$ ou OR (odds ratio)

† Incontinência urinária de esforço ou mista

* estatisticamente significativo considerando nível de significância de 0,05

IMC: índice de massa corporal; Ref: grupo de referência 
1,16 - 4,09). O teste de Hosmer-Lemeshow demonstrou que o modelo estava adequado aos dados $(p>0,05)$.

Os dados referentes aos comportamentos adotados na prática de exercício estão explanados na Tabela 3 e a caracterização e interferência da IU na Tabela 4 . O comportamento adotado pela maioria foi o ir à casa de banho antes do treino, seguindo-se o uso de penso absorvente. Das incontinentes, a maioria $(50,7 \%, \mathrm{n}=37)$ apresentava perdas no dia-a-dia e durante o exercício, e apenas uma minoria apresentava perdas exclusivamente no exercício $(9,6 \%$, $n=7$ ). A generalidade das mulheres apresentava uma IU leve a moderada, referindo perdas urinárias de quantidade pequena, com uma frequência igual ou inferior a uma vez por semana mas que interferia no seu dia-a-dia ou na prática de exercício.

Em relação à abordagem da IU nos ginásios, apenas $5,5 \%(n=16)$ afirmou que a temática foi abordada no momento da avaliação física inicial; $9,7 \%(n=28)$ reportou

Tabela 3 - Comportamentos adotados na prática de exercício físico

\begin{tabular}{lcc}
\hline & $\begin{array}{c}\text { Continentes } \\
\mathrm{n}=217\end{array}$ & $\begin{array}{c}\text { Incontinentes } \\
\mathrm{n}=73^{\dagger}\end{array}$ \\
\hline Modificação de exercícios & $4(1,9)$ & $9(12,7)$ \\
Não ingerir líquidos & $2(0,9)$ & $8(11,0)$ \\
Ir ao WC antes & $40(18,4)$ & $50(68,5)$ \\
Usar penso absorvente & $5(2,3)$ & $19(26,0)$ \\
\hline
\end{tabular}

Os dados são apresentados como contagem (\%)

† Foram incluídos todos os tipos de incontinência urinária que os exercícios foram orientados tendo em conta o fortalecimento do pavimento pélvico e a prevenção da IU numa aula de grupo; das 37 mulheres com PT, 13,5\% ( $n=5)$ afirmou que o mesmo aconteceu num treino individual. Cento e noventa e oito mulheres $(68,3 \%)$ já tinham ouvido falar dos PFMT.

\section{DISCUSSÃO}

Este estudo permitiu determinar uma prevalência de IU de $25,2 \%$ numa população de mulheres praticantes de exercício recreativo em ginásios, sendo a IUE a mais frequente. Estudos prévios, realizados em mulheres com idades compreendidas entre os 14 e os 83 anos, praticantes de exercício recreativo, apresentam valores variáveis para a prevalência de IU $(14,9 \%$ a $49,3 \%),{ }^{18,21-24}$ como observado na Tabela 5. Num estudo realizado com jovens nulíparas oriundas da mesma região do país que as do presente trabalho, a prevalência encontrada foi de $19,9 \%,{ }^{25}$ valor em linha com o obtido. Tendo por comparação estudos prévios realizados na população geral portuguesa, a prevalência de IU foi inferior à de $2019(35,2 \%)^{4}$ mas superior à de 2009 $(21,4 \%){ }^{3}$ Realce-se que neste último estudo, apenas foram incluídas mulheres com idade superior a 40 anos e, para a mesma faixa etária, verificámos uma prevalência de IU de $31,2 \%$.

Analisando a distribuição por idades, encontramos uma mediana semelhante entre mulheres continentes e incontinentes, e, no caso das incontinentes, $60,3 \%$ tem menos de

Tabela 4 - Caracterização da IU no dia-a-dia e na prática de exercício físico

\begin{tabular}{|c|c|c|c|c|}
\hline & \multicolumn{2}{|c|}{$\begin{array}{c}\text { Incontinentes } \\
\text { no dia-a-dia e no exercício } \\
n=37^{\dagger}\end{array}$} & \multirow{2}{*}{$\begin{array}{c}\text { Incontinentes } \\
\text { apenas no dia-a-dia } \\
n n=29^{\dagger}\end{array}$} & \multirow{2}{*}{$\begin{array}{c}\text { Incontinentes } \\
\text { apenas no exercício } \\
n=7^{\dagger}\end{array}$} \\
\hline & No dia-a-dia & No exercício & & \\
\hline \multicolumn{5}{|l|}{ Frequência } \\
\hline$\leq 1 \mathrm{vez} / \mathrm{semana}$ & $18(48,6)$ & $24(64,9)$ & $24(82,8)$ & $6(85,7)$ \\
\hline 2 ou 3 vezes/semana & $12(32,4)$ & $7(18,9)$ & $3(10,3)$ & $0(0)$ \\
\hline $1 \mathrm{vez} / \mathrm{dia}$ & $2(5,4)$ & $4(10,8)$ & $2(6,9)$ & $1(14,3)$ \\
\hline Várias vezes/dia & $5(13,5)$ & $2(5,4)$ & $0(0)$ & $0(0)$ \\
\hline Constantemente & $0(0)$ & $0(0)$ & $0(0)$ & $0(0)$ \\
\hline \multicolumn{5}{|l|}{ Quantidade } \\
\hline Pequena & $33(89,2)$ & $34(91,9)$ & $27(93,1)$ & $7(100)$ \\
\hline Moderada & $4(10,8)$ & $2(5,4)$ & $2(6,9)$ & $0(0)$ \\
\hline Grande & $0(0)$ & $1(2,7)$ & $0(0)$ & $0(0)$ \\
\hline \multicolumn{5}{|c|}{ Interferência no dia-a-dia ou exercícioł } \\
\hline Sim & $33(91,7)$ & $32(88,9)$ & $23(79,3)$ & $4(57,1)$ \\
\hline Não & $3(8,3)$ & $4(11,1)$ & $6(20,7)$ & $3(42,9)$ \\
\hline \multicolumn{5}{|c|}{ Gravidade (ICIQ-IU SF score) } \\
\hline Leve (1 a 5) & $17(45,9)$ & $21(56,8)$ & $19(65,5)$ & $5(71,4)$ \\
\hline Moderada (6 a 12) & $17(45,9)$ & $13(35,1)$ & $9(31)$ & $2(28,6)$ \\
\hline Grave (13 a 18) & $3(8,1)$ & $3(8,1)$ & $1(3,5)$ & $0(0)$ \\
\hline Muito Grave (19 a 21) & $0(0)$ & $0(0)$ & $0(0)$ & $0(0)$ \\
\hline
\end{tabular}

Os dados são apresentados como contagem (\%)

† Foram incluídos todos os tipos de incontinência urinária.

$\ddagger$ Independentemente do grau de interferência. 
Tabela 5 - Prevalência de IU

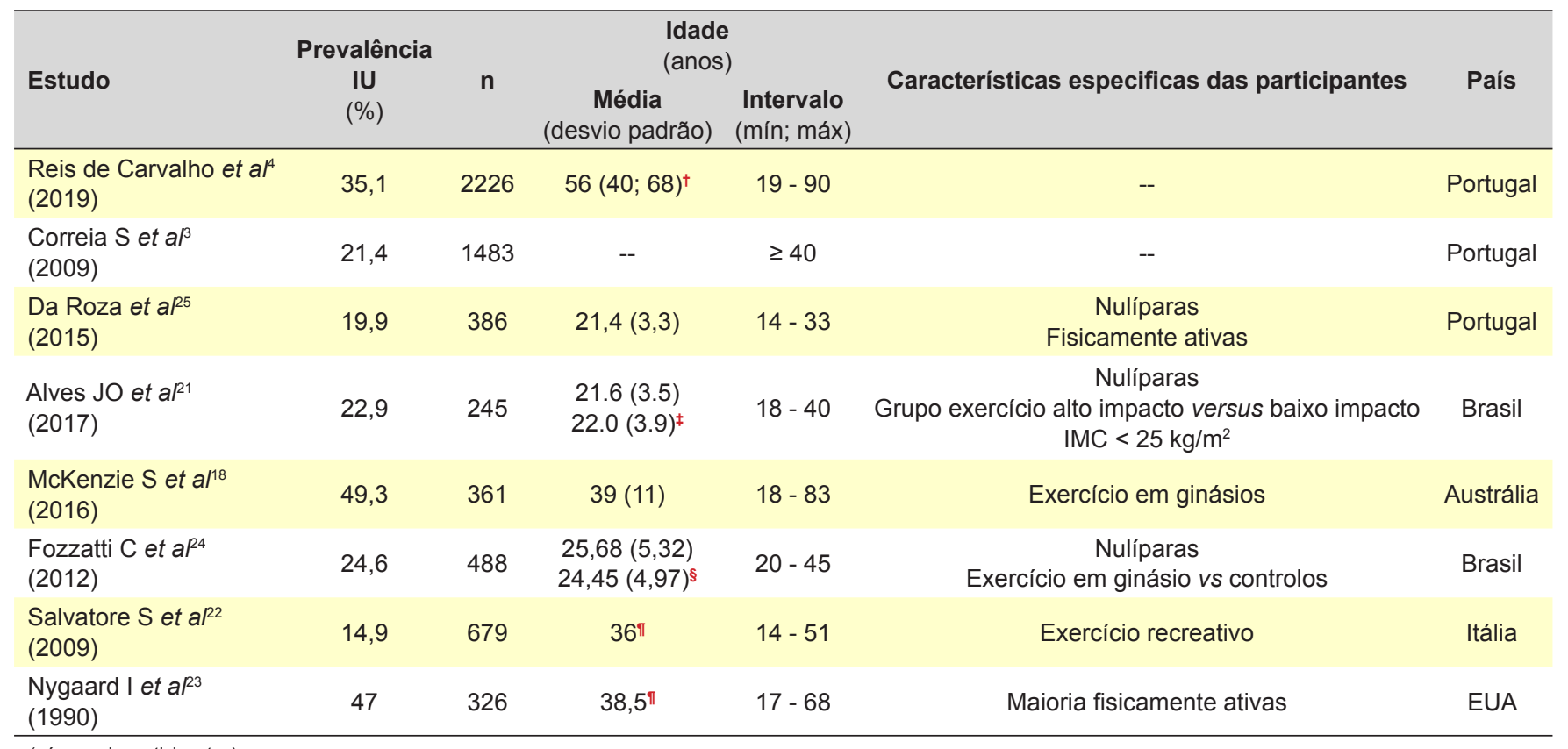

$\mathrm{n}$ (número de participantes).

† Representado por Mediana $\left(1^{\circ} \mathrm{Q} ; 3^{\circ} \mathrm{Q}\right)$

$\ddagger$ Dados referentes ao grupo alto impacto e grupo baixo impacto, respetivamente.

$\S$ Dados referentes ao grupo exercício em ginásios e ao grupo controlo, respetivamente.

Tा Dados sobre o desvio padrão não disponíveis

Mín: valor da idade mínima; máx: valor da idade máxima.

40 anos. A IU tem sido associada ao envelhecimento e à menopausa ${ }^{2,3,7,8}$ mas é expectável que analisando apenas mulheres fisicamente ativas, as idades sejam tendencialmente baixas. Fazendo a análise com base no escalão etário, o que apresenta maior prevalência de IU é o grupo entre os 40 e os 59 anos (32,4\%), enquanto que as perdas urinárias estavam presentes em $26,3 \%$ das mulheres com idade superior a 60 anos e em $22,4 \%$ daquelas com idade inferior a 40 anos. Na interpretação destes resultados temos de ter em consideração vários aspetos que podem ter levado a subestimar o número de mulheres incontinentes nas faixas etárias mais elevadas. Primeiro, esta temática pode ainda estar associada a um sentimento de vergonha, ${ }^{2,26}$ e talvez tal aconteça principalmente nas faixas etárias mais elevadas. Ficaram excluídas mulheres que abandonaram o ginásio devido à IU, e que potencialmente apresentavam uma IU de maior gravidade, o que pode explicar o fato de, na nossa amostra, a IU leve a moderada ser mais prevalente. Por último, como a amostra é menor nestes grupos, pequenas variações resultam num maior impacto na descrição percentual.

Vários estudos têm sido realizados, procurando perceber se existe associação entre exercício e IU. Na maioria, encontra-se uma considerável prevalência de IU, predominantemente associada às atividades de alto impacto ${ }^{27-}$ ${ }^{-29}$ (destacando-se saltos, corrida e exercícios que combinam movimentos abdominais e pélvicos), quer em atletas (prática desportiva organizada em contexto de competição), ${ }^{30-32}$ quer em praticantes de exercício recreativo. ${ }^{11,18,21-24,33,34}$ Por outro lado, outros trabalhos associam a IU ao sedentarismo. ${ }^{35-37}$ Dados sobre o efeito do exercício a longo prazo na função do pavimento pélvico e na IU são ainda escassos na literatura. ${ }^{29,38}$ Eliasson et a/ ${ }^{39}$ encontraram uma elevada prevalência de IU em ex-trampolinistas mas outros autores não constataram diferenças significativas entre atletas e controlos, ${ }^{41}$ ou entre praticantes de diferentes modalidades. ${ }^{40,41} \mathrm{Em}$ relação à maior frequência semanal $\left.\right|^{21,25,28,29,39} \mathrm{e}$ ao histórico temporal de prática desportiva, ${ }^{39}$ já tinha sido reportada uma associação positiva com a IU e o ICIQ score. ${ }^{30} \mathrm{Da}$ Roza et a ${ }^{25}$ constataram que as atletas (que semanalmente treinavam durante mais tempo, comparativamente às restantes participantes no estudo) apresentavam um risco 2,5 vezes superior de IU face a mulheres inativas. Contudo, nas praticantes de exercício recreativo não se verificou um incremento do risco relativo.

Neste trabalho, também não encontrámos associação estatisticamente significativa entre os parâmetros relacionados com o exercício e a IU (Tabela 2). Verificámos que $62,1 \%$ das mulheres continentes optou por treinos que incluem exercícios extenuantes ou de alto impacto. Este facto é concordante com a elevada prevalência de jovens na amostra, que tendencialmente realizam uma atividade física mais vigorosa. Porém, no grupo das mulheres incontinentes observámos uma percentagem mais reduzida $(50,9 \%)$ que realiza exercício extenuante em todas as faixas etárias. Podemos equacionar que as mulheres incontinentes alteraram os seus padrões de treino, desistindo de aulas mais extenuantes e optando por modalidades de baixo impacto, de forma a diminuir os episódios de perdas, ou terem mesmo abandonado completamente a prática desportiva. ${ }^{11,12,22,23}$

Em 1994, Nygaard et a ${ }^{\beta 2}$ sugeriu que para cada mulher 
existe um limiar de continência que quando excedido resulta na perda de urina, mesmo na ausência de qualquer fator de risco para IU. Numa recente revisão ${ }^{14}$, Nygaard e $\mathrm{B} \varnothing$, reafirmam esta teoria, acrescentando a hipótese de que o exercício extenuante pode ter um efeito prejudicial nas mulheres com um pavimento pélvico disfuncional, incapazes de realizar uma co-contracção inconsciente dos MPP atempadamente, mas um efeito positivo no fortalecimento muscular das mulheres com um pavimento pélvico funcional. A manobra de Knack (contração voluntária dos MPP antes e durante um aumento da PIA) demonstrou ser eficaz na prevenção da perda urinária com a tosse..$^{42,43}$ Esta técnica, de coordenação da pré contração com o exercício em execução, poderá ser aplicada em alguns treinos, mas será difícil naqueles com grande alternância de exercícios, repetições rápidas ou em número elevado.

A questão do aumento da PIA como fator predisponente a perdas urinárias também tem sido debatida e desconhece-se ainda o efeito concreto sobre os MPP. ${ }^{14}$ Alguns trabalhos demonstram que, durante exercícios como abdominais, esta não atinge em média valores mais elevados que a tosse ${ }^{44}$ ou o levantar da posição sentada, e há uma grande variabilidade inter-individual nos valores que em cada mulher são atingidos e tolerados. ${ }^{14} \mathrm{Um}$ outro estudo comparou a PIA durante exercícios 'normais' com planos alternativos considerados seguros para o pavimento pélvico e não encontrou diferenças significativas. ${ }^{45} \mathrm{Na}$ mesma revisão, ${ }^{14}$ os autores propõem que a PIA possa ter impacto nos MPP não pelo seu valor absoluto de carga sobre o pavimento pélvico mas por esta ser aplicada repetidamente e com carácter dinâmico.

Por último, desconhece-se o efeito da inclusão no plano de treino de modalidades de baixo impacto, numa perspetiva de 'contrabalanço' do efeito do exercício extenuante. Mulheres fisicamente ativas podem necessitar de um pavimento pélvico mais forte..$^{17,27}$ Os PFMT podem melhorar ou resolver os sintomas de todos os tipos de $\mathrm{IU},{ }^{46}$ e fazem parte do tratamento conservador, recomendado tanto para prevenção como primeira linha terapêutica, pela ICS e pela Sociedade Portuguesa de Ginecologia. ${ }^{2}$ Exercícios que possam fortalecer o core e os MPP, como Pilates e Yoga, têm sido explorados como potenciais alternativas, mas os dados ainda são insuficientes. ${ }^{47-50}$ De facto, $B \varnothing$ et al já tinha reportado uma taxa de IU nas professoras destas modalidades semelhante à da população geral, ainda que a maioria das participantes neste estudo praticava outras modalidades em simultâneo. ${ }^{51}$ Num trabalho de 2018, a autora em conjunto com Kruger et al, concluiu que os MPP apenas contraiam $30 \%$ a $50 \%$ de uma contração em contexto de PFMT, durante a ativação de outros grupos musculares (como abdominais e glúteos), pelo que se questiona a eficácia terapêutica e o impacto que as co-contrações podem ter nos MPP. ${ }^{52}$

Importa realçar a subjetividade e o que pode ter constituído uma fonte de viés para a classificação do tipo de exercício, como seja: a maioria das participantes realizava uma grande variedade de aulas com diferentes frequên- cias semanais; não foi contabilizado o tempo semanal de prática desportiva, apenas o número de treinos; não foram considerados os treinos de 'cardio' dada a ausência de discriminação da intensidade e tipologia de treino, assim como todas as respostas que não especificavam o tipo de exercício realizado; a categorização do treino de musculação foi baseada na autopercepção da intensidade das cargas utilizadas e não na avaliação objetiva das mesmas.

Em relação aos fatores de risco não relacionados com o exercício, encontramos uma associação positiva com a IU para a obstipação e o IMC $\geq 25 \mathrm{~kg} / \mathrm{m}^{2}$, o que vai ao encontro do descrito na literatura, que considera estas duas

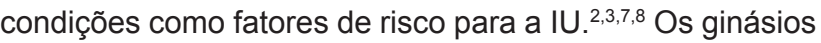
voltam a ter um importante papel neste contexto, não só através da promoção da atividade física mas também com serviços de aconselhamento nutricional e de hidratação. Programas não-cirúrgicos de perda de peso, em conjunto com alterações do estilo de vida, aparentam ter um modesto benefício na melhoria da IU nos primeiros três anos, ${ }^{53} \mathrm{e}$ um decréscimo de $5 \%$ a $10 \%$ do peso corporal demonstrou estar associado à diminuição dos episódios de perdas de urina em mulheres com excesso de peso. ${ }^{54}$ Também em relação à obstipação, o exercício pode ser uma opção de tratamento, apesar do seu efeito ainda não ter sido quantificado, ${ }^{55}$ aliado a uma dieta rica em fibras e a uma ingestão de fluidos adequada. ${ }^{56}$ De referir que a variável 'cirurgia genito-urinária' não foi incluída no modelo de regressão logística, apesar da sua associação significativa com a IU na análise univariada, uma vez que apresentava uma dimensão de amostra muito baixa, e tal poderia constituir um viés.

$\mathrm{Na}$ análise da associação entre a IU e potenciais fatores de risco (Tabela 1 e 2), devemos ter em consideração que o tamanho da amostra não foi calculado com esse objetivo, mas sim tendo por base a precisão desejada para estimativas de prevalência, o que pode ter contribuído para a ausência de diferenças estatisticamente significativas para alguns dos parâmetros analisados. Para além das limitações já referidas, não é possível estabelecer uma relação de causa-efeito, pois trata-se de um estudo transversal observacional e a data de início de sintomas para cada inquirida não foi determinada. Assim, não foi possível determinar se alguma das inquiridas já tinha tido perdas no passado (ainda que não nas últimas quatro semanas) e se os sintomas de IU começaram antes ou depois do início da prática de exercício físico.

As medidas comportamentais descritas na Tabela 3 já tinham sido identificadas em estudos prévios. ${ }^{11,18,22,23}$ Verificou-se que algumas mulheres adotaram comportamentos preventivos das perdas urinárias mesmo não tendo incontinência. Podemos concluir que mesmo sem incontinência há um receio nas mulheres de virem a ter algum episódio de perda urinária no futuro ou que algumas mulheres podem ter tido IU no passado, uma vez que o ICIQ-UI SF incide apenas nas últimas quatro semanas.

Menos de $30 \%$ das mulheres incontinentes tinha reportado este problema de saúde ao seu médico, o que está em concordância com o verificado por outros autores..$^{22,23,32}$ 
Esparza et al apontou a normalização da IU como uma das atitudes mais presentes entre as doentes, considerando este problema de saúde como algo inerente à idade e às características biológicas de ser mulher, principalmente nas mais velhas. ${ }^{9}$

Como demonstrado neste estudo a IU é raramente abordada nas avaliações físicas e sessões de treino em ginásio, o que pode contribuir para um sub-diagnóstico, sub-tratamento, para o abandono da atividade física e para a perpetuação da 'vergonha' e normalização associadas a este problema de saúde. A avaliação física e do estado de saúde, realizada na maioria dos ginásios no momento da iniciação da atividade e ao longo da sua prática, é uma oportunidade para o rastreio da IU mas também para educar para a saúde, prevenir e até porventura contribuir para o tratamento conservador da IU, promovendo-se a adoção de medidas de proteção do pavimento pélvico integradas no plano de treino.

Mais estudos são necessários para esclarecer a relação entre a IU e os vários tipos de exercício. Como trabalho futuro, seria interessante investigar a influência de um plano de PFMT em simultâneo com o exercício de alto impacto ou extenuante, assim como avaliar o conhecimento e sensibilidade dos treinadores para esta temática.

\section{CONCLUSÃO}

Este estudo demonstra que a IU é prevalente em muIheres praticantes de exercício físico recreativo em ginásio, sendo frequente não só nas idades mais avançadas, mas também nas faixas etárias mais jovens, e sem aparente relação com a frequência e intensidade de exercício físico praticado neste contexto. Verificou-se uma associação significativa da IU com a obstipação e o excesso de peso, ambos fatores de risco modificáveis. Esta temática raramente é abordada nos ginásios. Dada a elevada prevalência e alto impacto da IU na qualidade de vida, é necessário sensibilizar os profissionais e promover o seu papel ativo na identificação da IU, na abordagem da modificação dos seus fatores de risco e na implementação de estratégias preventivas no âmbito do exercício físico.

\section{AGRADECIMENTOS}

Os autores gostariam de agradecer a todas as mulheres que aceitaram participar neste estudo e aos ginásios que possibilitaram a recolha dos inquéritos.

\section{CONTRIBUTO DOS AUTORES}

MFS: Desenho do projeto; aquisição, análise e interpretação dos dados; escrita e revisão final do artigo.

RPC, CMO: Análise e interpretação dos dados; revisão final do artigo.

SM: Desenho do projeto; análise e interpretação dos dados; revisão final do artigo.

\section{PROTEÇÃO DE PESSOAS E ANIMAIS}

Os autores declaram que os procedimentos seguidos estavam de acordo com os regulamentos estabelecidos pelos responsáveis da Comissão de Investigação Clínica e Ética e de acordo com a Declaração de Helsínquia da Associação Médica Mundial actualizada em 2013.

\section{CONFIDENCIALIDADE DOS DADOS}

Os autores declaram ter seguido os protocolos do seu centro de trabalho acerca da publicação de dados.

\section{CONSENTIMENTO DO DOENTE}

Obtido.

\section{CONFLITOS DE INTERESSE}

Os autores declaram não ter conflitos de interesses relacionados com o presente trabalho.

\section{FONTES DE FINANCIAMENTO}

Este trabalho não recebeu qualquer tipo de suporte financeiro de nenhuma entidade no domínio público ou privado.

\section{REFERÊNCIAS}

1. Haylen BT, de Ridder D, Freeman RM, Swift SE, Berghmans B, Lee J, et al. An International Urogynecological Association (IUGA)/International Continence Society (ICS) joint report on the terminology for female pelvic floor dysfunction. Neurourol Urodyn. 2010;29:4-20.

2. Martins A, Candoso B, Negrao L, Alegra S. Consenso Nacional sobre Uroginecologia. Sociedade Portuguesa de Ginecologia - Secção de Uroginecologia. 2018. [consultado 2019 set 13]. Disponível em: http:// www.spginecologia.pt/academia/consensos/

3. Correia S, Dinis P, Rolo F, Lunet N. Prevalence, treatment and known risk factors of urinary incontinence and overactive bladder in the noninstitutionalized Portuguese population. Int Urogynecol J Pelvic Floor Dysfunct. 2009;20:1481-9.

4. Reis de Carvalho C, Costa N, Pereira I, Henriques A, Lourenço A, Calhaz-Jorge C. Incontinência urinária na mulher, um retrato da realidade portuguesa. Programa científico da $195^{\mathrm{a}}$ Reunião da Sociedade Portuguesa de Ginecologia. Lisboa: SPG; 2020 .

5. Mascarenhas T. Disfunções do pavimento pélvico: incontinência urinária e prolapso dos órgãos pélvicos. In: Oliveira CF, editor. Manual de Ginecologia (Vol. 2). Lisboa: Permanyer Portugal; 2011. p. 97-139.

6. Salvatore S, Rademakers K, DeLancey J, Igawa Y, Koelbl H, Laterza $\mathrm{RM}$, et al Pathophysiology of urinary incontinence, faecal incontinence and pelvic organ prolapse. In: Abrams $\mathrm{P}$, Cardozo L, Wagg A, Wein A, editors. Incontinence [e-book]. 6th ed. Bristol: ICI-ICS, International Continence Society; 2017.

7. Amaral MO, Coutinho EM, Nelas PA, Chaves CM, Duarte JC. Risk factors associated with urinary incontinence in Portugal and the quality of life of affected women. Int J Gynecol Obstet. 2015;131:82-6.

8. Milsom I, Altman D, Cartwright R, Lapitan MC, Nelson R, Sjöström $S$, et al. Epidemiology of urinary incontinence (UI) and other lower urinary tract symptoms (LUTS), pelvic organ prolapse (POP) and anal (AI) incontinence. In: Abrams P, Cardozo L, Wagg A, Wein A, editors. Incontinence [e-book]. $6^{\text {th }}$ ed. Bristol: ICl-ICS, International Continence Society; 2017. p. 1-141.

9. Esparza AO, Tomás MA, Pina-Roche F. Experiences of women and men living with urinary incontinence: a phenomenological study. Appl Nurs Res. 2018;40:68-75.

10. Lopes $M H$, Higa R. Restrições causadas pela incontinência urinária à vida da mulher. Rev Esc Enferm USP. 2006;40:34-41.

11. Brennand E, Ruiz-Mirazo E, Tang S, Kim-Fine S. Urinary leakage during exercise: problematic activities, adaptive behaviors, and interest in treatment for physically active Canadian women. Int Urogynecol J. 2018;29:497-503. 
12. Nygaard I, Girts T, Fultz NH, Kinchen K, Pohl G, Sternfeld B. Is urinary incontinence a barrier to exercise in women? Obstet Gynecol. 2005;106:307-14.

13. World Health Organization. Global recommendations on physical activity for health, 2010. [consultado 2019 set 13]. Disponível em: https://www. who.int/dietphysicalactivity/publications.

14. Bø K, Nygaard IE. Is physical activity good or bad for the female pelvic floor? A narrative review. Sports Med. 2020;50:471-84

15. Chisholm L, Delpe S, Priest T, Reynolds WS. Physical activity and stress incontinence in women. Curr Bladder Dysfunct Rep. 2019;14:174-9.

16. Bø K, Stien R. Needle EMG registration of striated urethral wall and pelvic floor muscle activity patterns during cough, Valsalva, abdominal, hip adductor, and gluteal muscle contractions in nulliparous healthy females. Neurourol Urodyn. 1994;13:35-41.

17. Bø K. Urinary incontinence, pelvic floor dysfunction, exercise and sport. Sports Med. 2004;34:451-64.

18. McKenzie S, Watson T, Thompson J, Briffa K. Stress urinary incontinence is highly prevalent in recreationally active women attending gyms or exercise classes. Int Urogynecol J. 2016;27:1175-84.

19. Tamanini JT, Dambros M, D'Ancona CA, Palma PC, Rodrigues Netto N Jr. Validation of the "International Consultation on Incontinence Questionnaire-Short Form" (ICIQ-SF) for Portuguese. Rev Saude Publica. 2004;38:438-44.

20. Klovning A, Avery K, Sandvik H, Hunskaar S. Comparison of two questionnaires for assessing the severity of urinary incontinence: the ICIQ-UI SF versus the incontinence severity index. Neurourol Urodyn. 2009;28:411-5.

21. Alves JO, Luz ST, Brandão S, Da Luz CM, Jorge RN, Da Roza T. Urinary incontinence in physically active young women: prevalence and related factors. Int J Sports Med. 2017;38:937-41.

22. Salvatore S, Serati M, Laterza R, Uccella S, Torella M, Bolis PF. The impact of urinary stress incontinence in young and middle-age women practising recreational sports activity: an epidemiological study. $\mathrm{Br} J$ Sports Med. 2009;43:1115-8.

23. Nygaard I, DeLancey JO, Arnsdorf L, Murphy E. Exercise and incontinence. Obstet Gynecol. 1990;75:848-51.

24. Fozzatti C, Riccetto C, Herrmann V, Brancalion MF, Raimondi M, Nascif $\mathrm{CH}$, et al. Prevalence study of stress urinary incontinence in women who perform high-impact exercises. Int Urogynecol J. 2012;23:1687-91.

25. Da Roza T, Brandão S, Mascarenhas T, Jorge RN, Duarte JA. Urinary incontinence and levels of regular physical exercise in young women. In J Sports Med. 2015;36:776-80

26. Newman DK, Cockerell R, Griebling TL, Rantell A, van Houten $P$, Palmer $\mathrm{MH}$. Primary prevention, continence promotion, models of care and education. In: Abrams P, Cardozo L, Wagg A, Wein A, editors. Incontinence [e-book]. $6^{\text {th }}$ ed. Bristol: ICI-ICS, International Continence Society; 2017. p. 2427-78.

27. Almousa S, Bandin van Loon A. The prevalence of urinary incontinence in nulliparous adolescent and middle-aged women and the associated risk factors: a systematic review. Maturitas. 2018;107:78-83.

28. Simeone C, Moroni A, Pettenò A, Antonelli A, Zani D, Orizio C, et al. Occurrence rates and predictors of lower urinary tract symptoms and incontinence in female athletes. Urologia. 2010;77:139-46.

29. de Mattos Lourenco TR, Matsuoka PK, Baracat EC, Haddad JM. Urinary incontinence in female athletes: a systematic review. Int Urogynecol J. 2018;29:1757-63.

30. Da Roza T, Brandão S, Mascarenhas T, Jorge RN, Duarte JA. Volume of training and the ranking level are associated with the leakage of urine in young female trampolinists. Clin J Sport Med. 2015;25:270-5.

31. Gram MC, Bø K. High level rhythmic gymnasts and urinary incontinence: prevalence, risk factors, and influence on performance. Scand $\mathrm{J}$ Med Sci Sports. 2020;30:159-65.

32. Nygaard IE, Thompson FL, Svengalis SL, Albright JP. Urinary incontinence in elite nulliparous athletes [published correction appears in Obstet Gynecol. 1994;84:342]. Obstet Gynecol. 1994;84:183-7.

33. Yang J, Cheng JW, Wagner H, Lohman E, Yang SH, Krishingner $G A$, et al. The effect of high impact crossfit exercises on stress urinary incontinence in physically active women. Neurourol Urodyn. 2019;38:749-56.

34. Poli de Araújo M, Brito LG, Rossi F, Garbiere ML, MaVilela ME, Bittencourt VF, et al. Prevalence of female urinary incontinence in crossfit practitioners and associated factors: an internet population- based survey. Female Pelvic Med Reconstr Surg. 2020;26:97-100.

35. Lee AH, Hirayama F. Physical activity and urinary incontinence in older adults: a community-based study. Curr Aging Sci. 2012;5:35-40.

36. Faleiro DJ, Menezes EC, Capeletto E, Fank F, Porto RM, Mazo GZ. Association of physical activity with urinary incontinence in older women: a systematic review. J Aging Phys Act. 2019;27:906-13.

37. Alhababi N, Magnus MC, Joinson C, Fraser A. A prospective study of the association between physical activity and lower urinary tract symptoms in parous middle-aged women: results from the Avon Longitudinal Study of Parents and Children. J Urol. 2019;202:779-86.

38. Shaw JM, Nygaard IE. Role of chronic exercise on pelvic floor support and function. Curr Opin Urol. 2017;27:257-61.

39. Eliasson K, Edner A, Mattsson E. Urinary incontinence in very young and mostly nulliparous women with a history of regular organised highimpact trampoline training: occurrence and risk factors. Int Urogynecol J Pelvic Floor Dysfunct. 2008;19:687-96.

40. Bø K, Sundgot-Borgen J. Are former female elite athletes more likely to experience urinary incontinence later in life than non-athletes?. Scand J Med Sci Sports. 2010;20:100-4

41. Nygaard IE. Does prolonged high-impact activity contribute to later urinary incontinence? A retrospective cohort study of female Olympians. Obstet Gynecol. 1997;90:718-22

42. Miller JM, Ashton-Miller JA, DeLancey JO. A pelvic muscle precontraction can reduce cough-related urine loss in selected women with mild SUI. J Am Geriatr Soc. 1998;46:870-4.

43. Miller JM, Sampselle C, Ashton-Miller J, Hong GR, DeLancey JO. Clarification and confirmation of the Knack maneuver: the effect of volitional pelvic floor muscle contraction to preempt expected stress incontinence. Int Urogynecol J Pelvic Floor Dysfunct. 2008;19:773-82.

44. Simpson S, Deeble M, Thompson J, Andrews A, Briffa K. Should women with incontinence and prolapse do abdominal curls?. Int Urogynecol J 2016;27:1507-12.

45. Tian T, Budgett S, Smalldridge J, Hayward L, Stinear J, Kruger J. Assessing exercises recommended for women at risk of pelvic floor disorders using multivariate statistical techniques. Int Urogynecol J. 2018;29:1447-54.

46. Dumoulin C, Cacciari LP, Hay-Smith EJ. Pelvic floor muscle training versus no treatment, or inactive control treatments, for urinary incontinence in women. Cochrane Database Syst Rev. 2018;10:CD005654

47. Dumoulin C, Adewuyi T, Booth J, Bradley C, Burgio K, Hagen S, et al. Adult conservative management. In: Abrams P, Cardozo L, Wagg A, Wein A, editors. Incontinence [e-book]. $6^{\text {th }}$ ed. Bristol: ICI-ICS International Continence Society; 2017. p. 1443-628.

48. Sha K, Palmer MH, Yeo S. Yoga's biophysiological effects on lower urinary tract symptoms: a scoping review. J Altern Complement Med. 2019;25:279-87.

49. Wieland LS, Shrestha N, Lassi ZS, Panda S, Chiaramonte D, Skoetz N Yoga for treating urinary incontinence in women. Cochrane Database Syst Rev. 2019;2:CD012668.

50. $\mathrm{B} \varnothing \mathrm{K}$, Herbert RD. There is not yet strong evidence that exercise regimens other than pelvic floor muscle training can reduce stress urinary incontinence in women: a systematic review. J Physiother. 2013;59:159-68.

51. Bø K, Bratland-Sanda S, Sundgot-Borgen J. Urinary incontinence among group fitness instructors including yoga and pilates teachers. Neurourol Urodyn. 2011:30:370-3.

52. Kruger J, Budgett D, Goodman J, Bø K. Can you train the pelvic floor muscles by contracting other related muscles? Neurourol Urodyn. 2019;38:677-83.

53. Yazdany T, Jakus-Waldman S, Jeppson PC, Schimpf MO, Yurteri-Kaplan LA, Ferzandi TR, et al. American Urogynecologic Society systematic review: the impact of weight loss intervention on lower urinary tract symptoms and urinary incontinence in overweight and obese women. Female Pelvic Med Reconstr Surg. 2020;26:16-29.

54. Wing RR, Creasman JM, West DS, Richter HE, Myers D, Burgio KL, et al. Improving urinary incontinence in overweight and obese women through modest weight loss. Obstet Gynecol. 2010;116:284-92.

55. Gao R, Tao Y, Zhou C, Li J, Wang X, Chen L, et al. Exercise therapy in patients with constipation: a systematic review and meta-analysis of randomized controlled trials. Scand J Gastroenterol. 2019;54:169-77.

56. Costilla VC, Foxx-Orenstein AE. Constipation: understanding mechanisms and management. Clin Geriatr Med. 2014;30:107-15. 